\title{
Ingeborg A umüller
}

\section{Dekolonisation und Nationwerdung in Sansibar}

Ifo-Institut für Wirtschaftsforschung München - Afrika-Studien 105

Weltforum Verlag, München-London 1980, 154 S., DM 44,-

Diese erweiterte und fortgeschriebene Buchfassung einer Münchener Dissertation von 1972 beschäftigt sich mit einem in der Afrikaforschung stark vernachlässigten Gebiet, dem vor allem seit der Union 1964 völlig im Schatten einer reichen Tansania-Literatur verbleibenden Zanzibar. Das macht das Buch auch dann zu einer Bereicherung der deutschen Literatur, wenn es auf weiten Gebieten nicht mehr ist als die konzise Auswertung der klassischen Darstellungen der Geschichte des islamischen Ost-Afrika (Trimingham, Prins) und der neueren politischen Entwicklung in Zanzibar (Lofchie).

Der Beitrag der Verfasserin zur Zansibar-Diskussion konzentriert sich auf die Herausarbeitung der ethnischen, von ihr als entscheidend angesehenen Hintergründe der Revolution von 1964, wobei allerdings das von ihr selbst wiedergegebene statistische Material (S. $115 \mathrm{ff}$.) eine so starke Verflechtung ethnischer Differenzierung und ökonomischer Stratifizierung der zanzibarischen Gesellschaft anzeigt, daß Lofchies These, beide Aspekte seien untrennbar verwoben (zitiert S. 114), überzeugender ist als die apodiktische Aussage der Verfasserin, es habe sich um einen ethnischen, nicht einen Klassenprotest gehandelt (S. 118).

Brun-Otto Bryde

\section{Wolfgang S. Freund}

\section{Welche Zukunft für den Iran?}

Aktuelle Zukunft Heft 1, W. Braumüller Universitätsverlagsbuchhandlung, Wien, 1981, 53 S., DM 12,80

Das Buch enthält eine kurze Biographie Abdolhassan Banisadrs, bietet dann eine kritische Analyse seiner französischen Schriften (2 Bücher und 9 Artikel als Primärquellen) und setzt sich schließlich mit seinem sozialen und entwicklungspolitischen Denken auseinander. Der Autor behandelt diesen Bereich unter den zwei Gesichtspunkten der Kernideen Banisadrs und seiner Reformentwürfe. Damit ist er der erste, der dieses Thema in der deutschsprachigen Fachliteratur aufbereitet. Die flüssige und konzise Diktion erlaubt das mühelose Verstehen seiner Studie - ein Positivum, das man dem Autor hoch anrechnen muß. - Der bibliographische und der Anmerkungsapparat eröffnen ein weites Feld an neuerer Literatur.

Erklärtes Ziel der Darstellung ist es, dem Leser einen Uberblick über das gegenwärtige "Entwicklungsdenken" von politischen Führern islamischer Länder zu vermitteln - ein Unterfangen, wofür sich stellvertretend das Schrifttum Banisadrs heranziehen lasse 
(S. 7). Dieses enthält - nach Meinung des Autors - möglicherweise Archetypen dessen, was "in vielen Köpfen von Staatslenkern der Dritten Welt heute vorgehen mag". Genauere Kenntnis davon könne uns, den "Entwickelten und Industrialisierten", den Umgang mit "diesen Ländern" erleichtern, ihn konstruktiver gestalten (S. 6).

Im folgenden beleuchtet Freund zentrale Denkansätze Banisadrs zur Olwirtschaft, über die Rolle der Armee und der Multinationalen und zur Stadtentwicklung. Was den Verfasser am meisten bei diesen von ihm sorgfältig geprüften Denkansätzen stört, ist die wiederholte Kritik Banisadrs an operationellen Elementen der Wirtschafts-, Sozial- und Außenpolitik Reza Pahlavis. Er vermißt einen pragmatischen Gegenvorschlag für die künftige Gestaltung iranischer Politik und Entwicklungsplanung (S. 15). Banisadr geißele rücksichtslos die gewesenen Zustände, ohne indessen sagen zu können, wie die neuen auszusehen hätten (S. 25, 33). Die analytische, sicherlich irgendwo auch pseudowissenschaftliche "Demontage des Gewesenen" scheine Banisadr mehr zu liegen als die Skizze eines "Masterplan" für den neuen, islamischen Iran (S. 15). Der Autor glaubt auch, einen Grund für diese Kritik gefunden zu haben: Man müsse sich klar werden darüber, wo es Banisadr primär darauf ankomme, in den Augen westlicher (d. h. pariserischer) Gönner und Förderer die Rolle des "anständigen, intellektualistischen, liberalen und linkslastigen besseren iranischen Menschen überzeugend zu spielen", und damit möge auch das o. g. Phänomen seiner "Negativ-Analyse" zusammenhängen (S. 30). Für den Autor enthält der Entwicklungsplan Banisadrs als Reformentwurf "wenig Originelles". Im Grunde beschränke sich sein Ansatz auf eine Forderung nach "umfassender Autarkie" (S. 13). Das abschließende Urteil Freunds über Banisadr, den er nach Paul Vieille als "piètre intellectuel" (S. 3, 17, 30) bezeichnet: Man werde gut beraten sein, Banisadrs soziales und entwicklungspolitisches Denken nicht gerade als "Jahrhundertentwurf" eines herausragenden Politikers der Dritten Welt zu werten. Zurückhaltung sei anzumelden bei dem Versuch, seine Schriften und Äußerungen als Paradestück eines neuen "Islamic Way of Development" hinzustellen (S. 17). Ein weiterer Einwand des Autors gegen Banisadrs Vorschläge: Er bemühe sich nicht, das "islamisch-schiitische Phänomen“ im Iran zu erklären und durchsichtiger zu machen (S. 3, 16, 35 f.). Viele seiner Schriften seien nicht authentisch (S. 30); die Formulierungen blieben derart pauschal und damit undeutlich, daß eine das Sachverständnis fördernde Aussage dabei eben nicht entstehe (S. 18). Die Feststellung des Autors: "Niemandem ist so recht klar, aufgrund welcher Meriten Banisadr iranischer Präsident geworden ist." Jener sei innen- wie außenpolitisch besehen ein "noch unbeschriebenes Blatt" (S. 3).

Meines Erachtens sind diese weitgehenden Folgerungen gewagt. Hier wird etwas Endgültiges über Banisadr konstatiert, ohne daß der Verfasser diese Feststellung auf eine hinreichend breite Basis stellen könnte: In seinem Vorwort räumt er selbst ein, er habe "verständlicherweise" nicht alle Schriften Banisadrs lesen können. Dessen zahlreiche muttersprachliche Aussagen sind nicht mit eingeflossen. "Natürlich würde die Konsultierung der persisch geschriebenen Texte manche Zuordnung erleichtern" (S. 3). Oder: "Am Thema tiefer Interessierte sollten sich die Mühe machen, den Stoff mit Hilfe der um vieles umfangreicheren persischen Schriften Banisadrs zu vertiefen" (S. 34). 
Auch scheint Freund keinen hinreichenden Kontakt zum unmittelbaren, historisch gewachsenen persischen Umfeld Banisadrs, geschweige denn zu diesem selbst, gehabt zu haben. Stattdessen brandmarkt er Banisadrs mangelhafte Beherrschung der französischen Sprache, die phonetisch "fremdartige" und syntaktisch "holprige" Ausdrucksweise (S. 6, 19, 34). Kurz gesagt, vermißt der Rezensent in der vorliegenden Studie gerade das, was für ein grundlegendes Verständnis unabdingbar wäre: die iranische Perspektive. Hier wird naturgemäß von einer europäischen Position aus analysiert und geurteilt, und der Leser sollte diese Verengung des Blickwinkels stets berücksichtigen - vor allem, wenn es um das "islamisch-schiitische Phänomen" geht (siehe dazu S. 3 f., 18, 33, 36).

Zwei wesentliche Züge bestimmen die Kritik des Autors an Banisadr. Zunächst kommt Freund zu einer im ganzen harten, aber nicht emotionsfreien Bewertung. Dagegen sucht er im weiteren Verlauf seine eigene Kritik auf dezente und sachlichere Art zu entschärfen. So wird etwa der Vorwurf des Autors, den Schriften Banisadrs mangele es am Pragmatismus, nun mit der Feststellung relativiert, daß einige seine Zeitungsartikel neues "Leben" in sich trügen - einfach deshalb, weil sie aus der empirischen Anschauung iranischer Geschehnisse geschrieben seien (S. 33; weitere Entschärfungen, S. 35, 37, 40 ff., 45).

Auch die Einstellung Freunds zum gestürzten Schah klärt sich nur nach und nach im Verlauf der Lektüre, denn eingangs wird er als ausgemachter Kenner der iranischen Verhältnisse - wenn auch ohne Werturteil - gezeichnet: "Vermutlich ist es überhaupt so, daß die Kategorien des im Iran Wirklichen von einem Mann wie Reza Pahlavi besser gesehen wurden als von Khomeiny und seinen Anhängern“ (S. 1). Erst später liest man, daß des Autors Meinung vom ehemaligen Kaiserlichen System so positiv denn doch nicht ist. Es stehe ihm völlig fern, dem zusammengebrochenen Schah-Regime "irgendeinen unangemessenen Lorbeer-Kranz posthum zu flechten ". Die Früchte der Pahlavi-Dynastie seien "verfault bis in die letzten Verästelungen des Baumes" (S. 29).

Nicht unproblematisch ist das Bestreben des Verfassers, Persönlichkeit und Ideen Banisadrs lediglich anhand von Selbstzeugnissen zu fixieren. Im guten Glauben hält er sich an dessen Veröffentlichungen. Doch wer die persische Mentalität kennt, dem wird rasch klar werden, wie flexibel die Iraner in ihren Äußerungen sind; diese Flexibilität tritt allerdings erst dann deutlich zutage, wenn die Theorie in die Praxis übergehen muß. Dementsprechend ist es - zugestanden - schwierig, einen Iraner nur von seinen Schriften

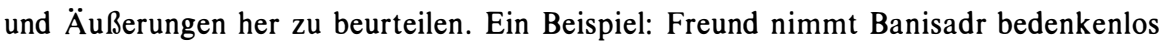
beim Wort, wenn dieser die (idealistische!) Forderung nach einer "umfassenden Autarkie" aufstellt (S. 4, 13 f., 35, 38). In Wirklichkeit aber sind sich Banisadr und andere iranische politische Führer, ohne es laut zu sagen, sehr wohl darüber im klaren, daß dieses Postulat keine realpolitischen Erfolgaussichten hätte. Was sie über kurz oder lang anstreben wollten, war in der Tat die Unabhängigkeit von den Großmächten. Generell wünschen sie solche internationalen Beziehungen, die gleichgewichtige Vorteile für beide Seiten bringen, d. i. nach dem Galtungschen Ansatz eine horizontale Interaktion ohne "spin-off"-Auswirkungen. Sie haben Angst vor einer Wirtschaftsbeziehung beispielsweise zu den USA. Denn diese würden bei jedem Handel mit dem Iran von einer Position 
der Stärke ausgehen können. Deshalb tritt der Iran lieber mit blockfreien und vornehmlich mit "Drittwelt-Ländern" (Indien, Pakistan, Nordkorea, Jugoslawien, Taiwan, Rumänien etc.) in Beziehung. Von daher ist es verständlich, wenn Banisadr in seinen Schriften selbst den Bereich der Kulturbeziehungen in allen seinen Facetten (Medien, Ausbildung, Auslandsstudium, Fremdsprachen etc.) "unbeleuchtet" läßt (S. 18, 41). Denn Banisadr befürchtet, wie auch die gegenwärtige iranische politische Führung, schon durch simple kulturelle Beziehungen eine gefährliche Handhabe für die Penetration Irans durch die Großmächte in allen Bereichen einzuräumen (also vom Galtungschen "spinoff-" zum "spill-over-"-Effekt). - Indes, der große Aufwand, mit dem der Iran trotz der finanziellen Misere nach wie vor seine im Westen studierenden Kinder unterstützt, belegt, daß weder Banisadr noch seine früheren Kollegen auf eine kulturelle Beziehung zu westlichen Ländern haben verzichten können - eine einseitige und stillschweigende Beziehung freilich. Auch hier zeigt sich wieder die oben bereits erwähnte Flexibilität des iranischen Politikers gegenüber dem Autarkie-Gedanken.

Wie steht es mit dem, was der Buchtitel verspricht? "Welche Zukunft für den Iran?" Wie erwartet, muß der Autor diese Frage offenlassen. Ausdrücklich verzichtet er auf die Skizzierung eines "Patentrezeptes" (S. 7); doch daß er nicht einmal mögliche Zukunftsmodelle für den Iran andeutet oder Entwicklungsperspektiven abwägt, muß als bedauerliche Schwäche seines Beitrages angesehen werden. Er spricht lediglich von einer Vernebelung "jeder sinnvollen Zukunftsprognose" und schließt dabei ninnenpolitische Uberraschungen" nicht aus (!). Diese seine Ratlosigkeit weiß er mit dem Weitertoben des irakisch-iranischen Krieges und mit dem Anhalten der islamistischen Ketzerjagd zu begründen (S. 43, 47).

Schließlich ist es auch auffällig, daß der Autor sich mit seinen Ausführungen zum Verfechter kapitalistischer Interessen macht, indem er nämlich Europa auf seine zukünftigen Chancen im Iran hinweist: Man genieße im Iran positive Vorurteile und moralischen Kredit. Bereiche solider Zusammenarbeit mit Europa würden dem Iran sicherlich das Gefühl geben, vor neuer Abhängigkeit von den USA sicher zu sein; und was noch mehr zähle: Er würde auch über eine Rückversicherung gegenüber der sowjetischen Bedrohung verfügen. "Hier stehen ... politische Türen offen, die es europäischerseits zu durchschreiten gilt" (S. 44, 46 f.).

Bei allen Vorbehalten muß man der Studie bescheinigen, daß sie dem Leser interessante und gut zusammengefaßte Informationen anbietet - Informationen, die wirklich von Belang für eine fundierte Meinungsbildung sind und die man anderswo oft nur durch zeitraubendes Suchen gewinnen kann. Die Schlußfolgerungen und Ansichten des Verfassers bieten, wie gezeigt, bisweilen Anlaß zum Widerspruch. Doch unbestritten hat Freund hier eine Darstellung vorgelegt, die der interessierte Leser als willkommene Anregung empfinden wird.

Darius Pourkian 